Editorial

\title{
Ginecologia oncológica como área de atuação
}

\section{Oncologic Gynecology as the Area of Activity}

\author{
Agnaldo Lopes da Silva Filho ${ }^{1}$ Jesus Paula Carvalho ${ }^{2,3}$ \\ ${ }^{1}$ Departamento de Ginecologia e Obstetrícia da Universidade Federal \\ de Minas Gerais, Belo Horizonte, MG, Brasil \\ 2 Disciplina de Ginecologia da Faculdade de Medicina da Universidade \\ de São Paulo, São Paulo, SP, Brasil \\ ${ }^{3}$ Serviço de Ginecologia Oncológica do Instituto do Câncer do Estado \\ de São Paulo, São Paulo, SP, Brasil \\ Rev Bras Ginec Obst 2016;38:1-3.
}

\section{Importância do Câncer Ginecológico no Brasil}

O câncer ginecológico tem uma alta incidência e é causa relevante de morbidade e óbitos no Brasil e no mundo. 0 Instituto Nacional do Câncer (INCA) estima a ocorrência de aproximadamente 30.000 novos casos por ano de câncer de colo uterino, corpo e ovário no Brasil. ${ }^{1}$ Esses tumores são responsáveis por pelo menos $10 \%$ de todas as neoplasias malignas nas mulheres brasileiras, exceto o câncer de pele não melanoma (- Tabela 1). A abordagem do câncer ginecológico é complexa e ampla compreendendo ações preventivas, diagnósticas e terapêuticas que podem ser realizadas por médicos generalistas, ginecologistas e obstetras e outros especialistas. Porém existem ações e procedimentos de alta complexidade que demandam conhecimentos e treinamentos específicos, que só podem ser assimilados através de programas de treinamento em situações e em Instituições que dispõem de recursos para fornecer estes treinamentos. Dessa forma, é fundamental que ginecologistas e obstetras possam se qualificar para prestar assistência em Ginecologia Oncológica de Alta Complexidade.

\section{Histórico e Definições da Ginecologia Oncológica no Mundo}

A European Society of Gynecologic Oncology (ESGO) define o Ginecologista Oncológico como um especialista em Ginecologia e Obstetrícia com treinamento e capacidade para: avaliar e abordar de forma abrangente pacientes com câncer ginecológico ou de mama. Na União Europeia, o ginecologista habitualmente conduz o tratamento para câncer de mama, com exceção da Dinamarca, Finlândia, Irlanda, Holanda e Reino Unido; conduzir tratamento clínico e/ou cirúrgico das neoplasias malignas do trato genital feminino e mama (procedimentos cirúrgicos complexos abdominais) e praticar Ginecologia Oncológica em uma instituição em que estejam disponíveis todas as modalidades para tratamento oncológico, incluindo ainda procedimentos diagnósticos, terapêuticos e seguimento das pacientes.

A Ginecologia Oncológica é uma especialidade reconhecida nos Estados Unidos desde 1969 o que resultou em uma melhora significativa dos resultados nas mulheres com câncer ginecológico. ${ }^{2}$ Esse profissional deve conduzir tratamento clínico e/ou cirúrgico das neoplasias malignas do trato genital feminino e praticar Ginecologia Oncológica em um contexto multidisciplinar. A sua formação deve ser direcionada para o câncer ginecológico, com conhecimentos específicos sobre a fisiopatologia, biologia tumoral, patologia, radioterapia, quimioterapia e cuidados paliativos. Esse profissional necessita um treinamento direcionado para aquisição de habilidades cirúrgicas avançadas.

A duração da formação em Ginecologia Oncológica é de três anos nos Estados Unidos e Reino Unido e de quatro anos na Alemanha. Segundo a ESGO, a duração da formação de subespecialidade deve incluir um mínimo de dois anos em um programa aprovado e deve incluir atividades clínicas e de pesquisa nas seguintes áreas: treinamento cirúrgico em unidade de oncologia, treinamento de Cirurgia Geral, Urologia, Radioterapia, Oncologia Clínica, Patologia e Citopatologia, Psico-oncologia e Biologia Tumoral. Exige ainda um número mínimo de cirurgias para obtenção do título na subespecialidade: 30 casos de cirurgia para câncer de endométrio, ovário ou tuba uterina; quinze casos de histerectomia radical; cinco casos de cirurgias para outras malignidades e pelo menos cinco casos de vulvectomia com linfadenectomia.

\footnotetext{
Address for correspondence Agnaldo Lopes da Silva Filho, PhD, Departamento de Ginecologia e Obstetrícia da Faculdade de Medicina da UFMG, Avenida Professor Alfredo Balena 190 -Santa Efigênia, Belo Horizonte, MG, Brasil CEP: 30130-100 (e-mail: agnaldo. ufmg@gmail.com).
}

Dol http://dx.doi.org/ 10.1055/s-0035-1570106. ISSN 0100-7203.
Copyright (c) 2016 by Thieme Publicações License terms Ltda, Rio de Janeiro, Brazil 
Tabela 1 Incidência dos cânceres ginecológicos no Brasil Estimativa 2014

\begin{tabular}{|l|l|l|}
\hline${\text { Localização } \mathbf{1}^{\mathbf{a}}}$ & Casos Novos & $\%$ \\
\hline Colo do útero & 15.590 & 5,7 \\
\hline Corpo do útero & 5.900 & 2,2 \\
\hline Ovário & 5.680 & 2,1 \\
\hline Total & 27.170 & 10 \\
\hline Fonte: INCA & \\
\hline
\end{tabular}

\section{Ginecologia Oncológica no Programa de Residência Médica de Ginecologia e Obstetrícia no Brasil}

O treinamento em Ginecologia Oncológica é exigente, demanda tempo, recursos e dedicação em centros especializados. Novas tecnologias são incorporadas constantemente e novos paradigmas são incorporados aos protocolos de maneira cada vez mais rápida. O tempo de treinamento é muito maior do que as poucas semanas de treinamento específicos inseridas nos programas de residência médica em Obstetrícia e Ginecologia, que se mostram insuficientes para que os egressos possam prestar uma assistência adequada às mulheres com câncer ginecológico.

A inexistência de uma área de atuação em Ginecologia Oncológica de Alta Complexidade impossibilita a criação de programas de residência específicos nesta área com um a dois anos de duração. Com exceção da Ginecologia e Obstetrícia, todas as áreas básicas, incluindo a Clínica Médica, Cirurgia Geral e Pediatria, estão representadas na Cancerologia com a suas respectivas subespecialidades. A residência médica é a melhor forma de treinamento em especialidades, principalmente em áreas com vários níveis de complexidade e combinação de conhecimentos clínicos e cirúrgicos. Este é precisamente o caso da Ginecologia Oncológica de Alta Complexidade. A residência médica pode propiciar uma uniformidade na formação dos profissionais e consequentemente melhores resultados para as pacientes.

\section{Ginecologia Oncológica como Área de Atuação}

A Ginecologia Oncológica é uma especialidade reconhecida em praticamente todos os países que apresentam uma assistência médica de qualidade, com exceção do Brasil, México e Rússia. De forma geral as três áreas de atuação mais reconhecidas são a Ginecologia Oncológica, seguida pela Medicina Fetal, Reprodução Humana e Uroginecologia. A - Tabela 2 mostra as subespecialidades reconhecidas nos EUA, Alemanha e Reino Unido, países com um padrão de Medicina de alto nível. No Brasil a situação é bem divergente, a Associação Médica Brasileira (AMB) reconhece como áreas de atuação em Ginecologia e Obstetrícia a Densitometria Óssea, Endoscopia Ginecológica, Mamografia, Medicina Fetal, Reprodução Humana, Sexologia e Ultrassonografia em
Tabela 2 Áreas de atuação reconhecidas nos EUA, Alemanha, Reino Unido e Brasil

\begin{tabular}{|c|c|c|c|c|}
\hline $\begin{array}{l}\text { Áreas } \\
\text { de atuação }\end{array}$ & EUA & Alemanha & $\begin{array}{l}\text { Reino } \\
\text { Unido }\end{array}$ & Brasil \\
\hline $\begin{array}{l}\text { Ginecologia } \\
\text { oncológica (GO) }\end{array}$ & $\swarrow$ & $\nu$ & $\nu$ & \\
\hline $\begin{array}{l}\text { Medicina } \\
\text { materno-fetal }\end{array}$ & $\boldsymbol{V}$ & $v$ & レ & レ \\
\hline $\begin{array}{l}\text { Medicina } \\
\text { reprodutiva }\end{array}$ & $\boldsymbol{V}$ & $\boldsymbol{V}$ & レ & レ \\
\hline Uroginecologia & $\boldsymbol{V}$ & 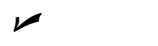 & レ & \\
\hline $\begin{array}{l}\text { Densitometria } \\
\text { óssea }\end{array}$ & & & & $\nu$ \\
\hline $\begin{array}{l}\text { Endoscopia } \\
\text { Ginecológica }\end{array}$ & & & & $\nu$ \\
\hline Mamografia & & & & 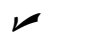 \\
\hline Sexologia & & & & 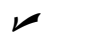 \\
\hline $\begin{array}{l}\text { Ultrassonografia } \\
\text { em GO }\end{array}$ & & & & $\nu$ \\
\hline
\end{tabular}

Ginecologia e Obstetrícia. O objetivo de se criar áreas de atuação consiste na necessidade de formação complementar em áreas complexas cuja curva de aprendizado não se conclui durante a residência médica em áreas básicas. As áreas de atuação quase sempre podem ser exercidas por mais de uma especialidade. No caso da Ginecologia Oncológica, considera-se a cancerologia cirúrgica uma especialidade afim para essa área de atuação.

A Ginecologia Oncológica tem vários níveis de complexidade durante o processo de diagnóstico e tratamento. ${ }^{3,4}$ Houve uma melhora significativa dos resultados, incluindo sobrevida, nas pacientes com câncer de colo uterino, endométrio, vulva e principalmente câncer de ovário, tratadas por um ginecologista oncológico. ${ }^{3,5-8}$ Tem ocorrido um movimento na última década pela centralização do tratamento do câncer em centros especializados. Isto vem a partir do reconhecimento de que o atendimento multidisciplinar, incluindo o acesso às avaliações do ginecologista oncológico, radioterapêuta e oncologista clínico, pode melhorar os resultados dos pacientes. ${ }^{3,4,9,10}$

\section{Considerações Finais}

Ressaltamos a necessidade de garantir que o médico ginecologista e obstetra, mantenha a sua atuação no rastreamento das neoplasias e condução do tratamento dos casos de menor complexidade. A - Tabela 3 mostra um sumário das razões para criação da área de atuação em Ginecologia Oncológica. Após reconhecimento da área de atuação em Ginecologia serão definidos os critérios para certificação, assim como a adoção de regras transitórias para os profissionais que já atuam nessa área. A criação da área de atuação vai possibilitar oferecer anos adicionais de residência médica em Ginecologia e Obstetrícia com atuação em Ginecologia Oncológica de Alta Complexidade. Longe de ser uma atitude excludente, a Ginecologia Oncológica 
Tabela 3 Razões para criação da área de atuação em Ginecologia Oncológica

\begin{tabular}{|l|l|}
\hline $\mathbf{1}$ & $\begin{array}{l}\text { O câncer ginecológico é muito prevalente: atinge aproximadamente } 30.000 \text { brasileiras a cada ano (10\% de todas as } \\
\text { neoplasias malignas no sexo feminino); }\end{array}$ \\
\hline $\mathbf{2}$ & $\begin{array}{l}\text { A abordagem do câncer ginecológico é complexa e requer um profissional capacitado, com conhecimentos específicos } \\
\text { sobre a fisiopatologia, biologia tumoral, patologia, radioterapia e quimioterapia; além de habilidades cirúrgicas } \\
\text { avançadas; }\end{array}$ \\
\hline $\mathbf{3}$ & $\begin{array}{l}\text { A Ginecologia Oncológica consiste em uma área de atuação extremamente consolidada há mais de } 45 \text { anos, sendo } \\
\text { reconhecida em praticamente todos os grandes países. As áreas de atuação em Ginecologia e Obstetrícia reconhecidas } \\
\text { pela Associação Médica Brasileira (AMB) são absolutamente discrepantes com os outros países e não inclui a } \\
\text { Ginecologia Oncológica; }\end{array}$ \\
\hline $\mathbf{4}$ & $\begin{array}{l}\text { O treinamento durante a residência médica em Obstetrícia e Ginecologia é insuficiente para prestar uma assistência } \\
\text { adequada às mulheres com câncer ginecológico. O não reconhecimento da Ginecologia Oncológica representa uma } \\
\text { enorme barreira para a formação complementar desses profissionais; }\end{array}$ \\
\hline $\mathbf{5}$ & \begin{tabular}{l} 
Mulheres com câncer ginecológico assistidas por um Ginecologista Oncológico apresentam melhor prognóstico. \\
\hline
\end{tabular}
\end{tabular}

de Alta Complexidade pretende promover os mecanismos para que ginecologista e obstetras de todo o pais adquiram qualificação adequada para atuar em toda a cadeia de eventos que envolve a prevenção, o diagnóstico e o tratamento do câncer ginecológico, inclusive os procedimentos de alta complexidade.

\section{Referências}

1 Brasil. Ministério da Saúde. Instituto Nacional de Câncer José Alencar Gomes da Silva [Internet]. Vigilância do câncer e seus fatores de risco. Rio de Janeiro: INCA; 2014 [citado 2014 Ago 1]. Disponível em: <http://www2.inca.gov.br/wps/wcm/connect/ acoes_programas/site/home/nobrasil/programa_epidemiologia_vigilancia/>

2 Averette HE, Wrennick A, Angioli R. History of gynecologic oncology subspecialty. Surg Clin North Am 2001;81(4):747-751

3 Soegaard Andersen E, Knudsen A, Svarrer T, et al. The results of treatment of epithelial ovarian cancer after centralisation of primary surgery. Results from North Jutland, Denmark. Gynecol Oncol 2005;99(3):552-556
4 Woo YL, Kyrgiou M, Bryant A, Everett T, Dickinson HO. Centralisation of services for gynaecological cancers - a Cochrane systematic review. Gynecol Oncol 2012;126(2):286-290

5 Giede KC, Kieser K, Dodge J, Rosen B. Who should operate on patients with ovarian cancer? An evidence-based review. Gynecol Oncol 2005;99(2):447-461

6 Gagliardi AR, Fung MF, Langer B, Stern H, Brown AD. Development of ovarian cancer surgery quality indicators using a modified Delphi approach. Gynecol Oncol 2005;97(2):446-456

7 Reade C, Elit L. Trends in gynecologic cancer care in North America. Obstet Gynecol Clin North Am 2012;39(2):107-129

8 Roland PY, Kelly FJ, Kulwicki CY, Blitzer P, Curcio M, Orr JW Jr. The benefits of a gynecologic oncologist: a pattern of care study for endometrial cancer treatment. Gynecol Oncol 2004;93(1): 125-130

9 National Cancer Institute [Internet]. SEER Cancer Statistics Review, 1975-2011. 2013 [cited 2014 Ago 1]. Available from: <http://seer.cancer.gov/csr/1975_2011/sections.html>

10 Geomini PM, Kruitwagen RF, Bremer GL, Massuger L, Mol BW. Should we centralise care for the patient suspected of having ovarian malignancy? Gynecol Oncol 2011;122(1):95-99 